\title{
Radar Micro-Doppler Feature Extraction Using the Spectrogram and the Cepstrogram
}

\author{
R. I. A. Harmanny \\ Thales Nederland B.V. \\ Delft, The Netherlands \\ ronny.harmanny@nl.thalesgroup.com
}

\author{
J. J. M. de Wit \\ Department of Radar Technology \\ TNO \\ The Hague, The Netherlands \\ jacco.dewit@tno.nl
}

\author{
G. Prémel Cabic \\ Thales Nederland B.V. \\ Delft, The Netherlands \\ gilles.premelcabic@nl.thalesgroup.com
}

\begin{abstract}
The radar micro-Doppler signature of a target is determined by parts of the target moving or rotating in addition to the main body motion. The relative motion of parts is characteristic for different classes of targets, e.g. the flapping motion of a bird's wings vs. the spinning of propeller blades. In the present study, the micro-Doppler signature is exploited to discriminate birds and small unmanned aerial vehicles (UAVs). Emphasis is on micro-Doppler features that can be extracted from spectrograms and cepstrograms, enabling the human eye or indeed automatic classification algorithms to make a quick distinction between man-made objects and bio-life. In addition, in case of man-made objects, it is desired to further characterize the type of mini-UAV to aid the threat assessment. Also this characterization is done on the basis of micro-Doppler features.
\end{abstract}

Keywords-radar, time-frequency analysis, birds, mini-UAVs, classification, cepstrum

\section{INTRODUCTION}

Within the security and defense domain, radar is more and more applied in the confined and crowded urban and littoral environments. Consequently, there is a demand for detecting and classifying a wider range of small targets such as mopeds, dismounts, animals, birds, flocks of birds, and mini-drones. Basically, detection of these smaller targets requires lowering the detection threshold, with respect to both target radar cross section (RCS) and Doppler velocity. However, the sheer number of objects in crowded littoral and urban environments may potentially saturate the radar signal processing, leading to, e.g., lost tracks. Ultimately, situational awareness is affected.

In these environments, full situational awareness can be maintained only if target classification can be done reliably and rapidly. Rapid classification allows filtering-out objects that are irrelevant for the current mission. For this first rapid classification, distinction between broad target classes may be sufficient. Depending on the mission, these broad classes could be man-made object, i.e., a potential threat, and bio-life, i.e., a non-threat, such as a bird. In a next classification step, it is desired to provide further separation within the potential threat classes to aid the threat assessment. For instance, the size or number of rotors of a drone may be an indication of its maximum payload. In this paper, the potential of exploiting
micro-Doppler properties for this two-step classification approach will be reviewed.

The classification problem addressed within the current study focuses on recognizing small unmanned aerial vehicles (UAVs). Mini-UAVs are an emerging threat and exhibit so called 'LSS' characteristics, for Low (altitude), Small (RCS) and Slow (speed), which makes them challenging radar targets when they operate in an environment with for instance birds.

To reduce the number of false alarms, it is important to quickly classify a UAV as a man-made object, preferable before the tracking stage where the identity of all objects currently present is maintained, and thus the number of uninteresting objects should be minimal to prevent track overload. In the next step, further characterization of the UAV is desired. Some characteristics of interest are the type of UAV, the number of rotors, approximate size, etc. This classification can be done by a trained human operator just by visual inspection of the preprocessed measurement data. It is also possible to use automatic recognition. Key is that the data is measured and presented such that certain features can be extracted that - combined - characterize the target class. A simple Bayesian classifier can e.g. be used to perform automatic classification. An important advantage of this approach is that this type of classifier also works with a subset of available features, in case some features are unstable or of low quality.

This paper discusses only a part of the work done in a much broader research project in which different approaches for classification are investigated [1]. Here, the emphasis is on micro-Doppler features that enable fast distinction between birds and mini-UAVs and that can be derived from spectrograms and cepstrograms in a rather straightforward manner.

Potentially relevant micro-Doppler features are discussed in Section II. Section III and IV discuss the spectrogram and cepstrogram respectively as the two important sources of features. Section V shows two real measurement examples. Sections VI presents the conclusion. 


\section{RELEVANT FEATURES}

Suitable features provide information on target parameters and are discriminative between relevant target classes. For reliable classification under varying conditions, features must be robust with respect to target type, radar settings, and measurement parameters, such as carrier frequency, sample rate, polarization, and aspect angle. In this section, the relevant micro-Doppler features for classification of birds and miniUAVs are reviewed. Some explanatory examples of spectrograms of real and synthetic targets, both man-made and bio-life, can be found in Chen [2]. His work is also a good starting point for understanding the micro-Doppler phenomenon in general, feature extraction, classification and related micro-Doppler concepts.

\section{A. Radar Cross Section}

In general, the RCS of mini-UAVs is higher than that of birds (depending on the species). RCS is therefore a discriminative feature, but it is not a robust feature. Observed RCS levels fluctuate significantly as function of aspect angle and radar frequency. One reason is that small and mediumsized birds and mini-UAVs are in the Mie resonance region for $\mathrm{S}$-band and $\mathrm{X}$-band radar frequencies.

\section{B. Main Velocity Component}

Typically, the main velocity component in a spectrogram is due to the motion of a target as a whole, assuming the body or fuselage gives the strongest reflection. Velocity components due to moving parts are usually weaker. The velocity of birds and mini-UAVs is in the same range, such that radial velocity is not discriminative. Also, radial velocity is not a robust feature since it depends on direction of flight.

\section{Spectrogram Periodicity}

Many target micro-motions are of periodic nature, i.e., the pendulum-like motion of a bird's wings and the rotation of rotor blades. The related spectrograms are periodic as well. The period of a mini-UAV spectrogram is determined by the rotor rotation rate, which is generally one order of magnitude higher than that of manned helicopters. The period of a bird spectrogram is related to the wing beat cycle. The wing beat frequency of birds is between 2 and 20 beats per second, depending on the species.

\section{Spectrum Width}

The spectrum width indicates the maximum velocity of the micro-motions relative to the main velocity component. This feature can be exploited to distinguish birds from mini-UAVs. The spectrum width associated with flying birds is just several meters per second. Typically, the velocity spread related to spinning rotors is much wider. The spectrum width feature is robust with respect to radar parameters, but it depends on the target aspect angle. High sampling rates are necessary to obtain the unambiguous Doppler spectrum.

\section{E. Spectrogram Symmetry}

The shape of the spectrogram relative to the main velocity component is indicative for the number of moving parts and their relative direction of motion. Typically, the spectrogram of a rotor with an even number of blades is symmetric about the main velocity component; the blades give rise to the 'blade flash' at the same time. In case of a rotor with an odd number of blades, the flashes occur after each other resulting in an asymmetric spectrogram (for some examples see, e.g., [3]). Also birds induce an asymmetric spectrogram, because both wings move up and down at the same time.

\section{SPECTROGRAM GENERATION}

A spectrogram reveals the instantaneous spectral content of the time-domain signal and also the variations of the spectral content over time. The appearance of a spectrogram depends on waveform and processing parameters, which will be discussed next.

A spectrogram is obtained by taking the magnitude squared of the short time Fourier transform (STFT) of a discrete signal, where the STFT can be written as:

$$
\operatorname{STFT}\{x[n]\} \equiv X(m, k)=\sum_{n=-\infty}^{\infty} x[n] w[n-m] e^{-j 2 \pi k n / N}
$$

Here, $x[n]$ is the discrete signal, $w[n]$ is the discrete window function, which is nonzero in $[0 . . N]$ and zero elsewhere, $N$ is the number of samples in the analysis window, and $k$ is the discrete frequency $k \omega_{0}$, where $\omega_{0}$ is the Fourier resolution, $\omega_{0}=2 \pi f_{s} / N$ with sampling frequency $f_{s}$. For radars measuring range and Doppler, $f_{s}$ is sometimes called PRF or SRF for pulse and FMCW-radars respectively. Index $m$ determines the position of the analysis window. By repeatedly calculating the STFT with increasing $m$ using a step size $\Delta m$, the spectrogram can be obtained. $\Delta m$ can be chosen such to realize a certain overlap between two consecutive analysis windows, which gives a smoother result in the time dimension. Evidently, for $N$, a power of 2 can be chosen or $N$ can be zeropadded to a power of 2 in order to enable using the FFT which is computationally faster compared to (1). We will assume this is the case in the remainder of this text.

If we want to generate a visually useful spectrogram of a certain event, such as the flapping of a bird's wing, we first need to choose a value for $N$. As a rule of thumb, we should choose to have the integration interval $N / f_{S}$ as a fraction of the length of the event. This we refer to as the 'short integration interval'. Note that the radar wavelength is critical in order to obtain a certain velocity resolution $\Delta v$, as, $\Delta v=\frac{\lambda}{2 T}=\frac{\lambda f_{s}}{2 N}$. To quantify the example, the wing beat frequency of the majority of bird species is in the range of 2 to 20 beats per second, and the maximum radial velocity around $15 \mathrm{~m} / \mathrm{s}$ depending on the measurement angle. If we measure with $\mathrm{X}$-band, then $\lambda \approx$ $3 \mathrm{~cm}$. If we take a fairly high wing beat frequency of $10 \mathrm{~Hz}$ and sample it 5 times faster, i.e. $50 \mathrm{~Hz}$, our $N / f_{s}$ becomes $20 \mathrm{~ms}$. This fixes $\Delta v$ to $0.75 \mathrm{~m} / \mathrm{s}$, which is an acceptable value. The spectrum width associated with flying birds is only several meters per second. The sample rate can thus be relatively low, i.e., several $\mathrm{kHz}$ in $\mathrm{X}$-band

Rotor blades exhibit much fasters events, with much higher velocities. The event - a single rotation of a rotor - is usually in the order of several milliseconds with blade tip velocities reaching up to $200 \mathrm{~m} / \mathrm{s}$ or higher. With a $\mathrm{CW}$ radar we can realize the high $f_{s}$ required to measure the high velocity, say 
several tens of $\mathrm{kHz}$. Such high sample rates are also feasible for some short-range surveillance radars.

For periodic events that are 'stable' for a while, we can generate the 'long integration interval' spectrogram. By applying a longer integration time $N / f_{s}$, several cycles of the rotor blades are included. The instantaneous spectral content is now dominated by the rotation rate of the blades causing modulation peaks. The instantaneous radial velocity of the blades is no longer observable. The difference between short and long integration interval is shown in Fig. 1.
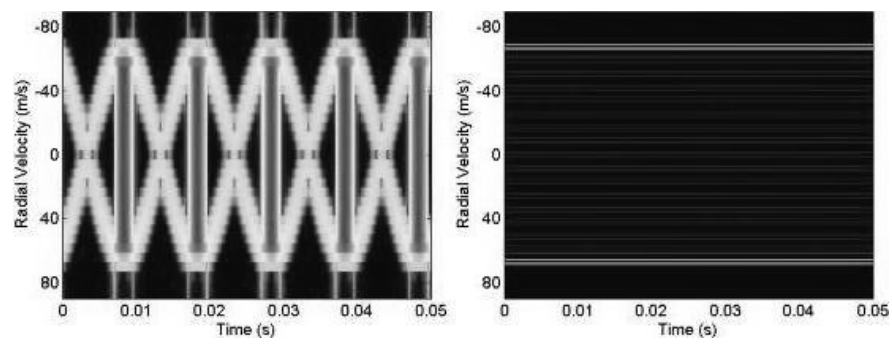

Fig. 1. Simulated spectrograms of rotating helicopter blades, using a relatively short integration time of $2.5 \mathrm{~ms}$ (left) and a relatively long integration time of $150 \mathrm{~ms}$ (right).

In Fig. 2 the effect of overlapping integration intervals is shown. In the left figure the overlap is only $40 \%$. The discontinuities in frequency from one integration interval to the next are apparent. In the right figure the overlap is $80 \%$. Here the changes of the spectral content are more gradual and the spectrogram is more detailed.
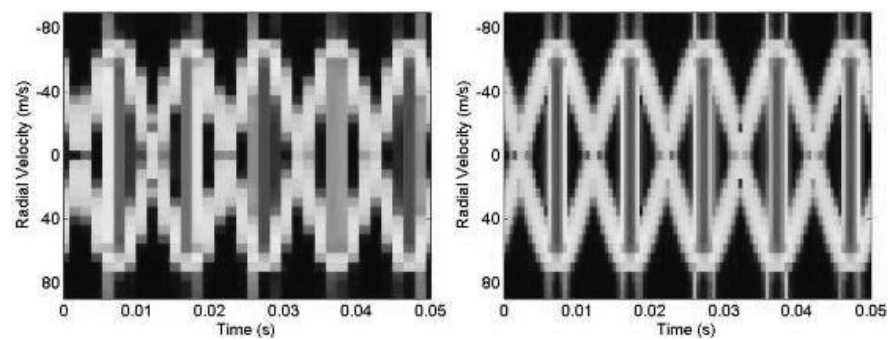

Fig. 2. Simulated spectrograms of rotating helicopter blades, using different overlaps between successive time sequences: left $40 \%$ and right $80 \%$.

\section{CEPSTROGRAM GENERATION}

The power cepstrum was defined in a 1963 paper by Bogert et al [4]. One can use it to obtain information about the rate of change in the spectrum and it has been used for e.g. characterizing seismic echoes from earthquakes and for human speech analysis. In the original paper the (continuous) cepstrum is defined as,

$$
\equiv\{f(t)\}=\left|\mathcal{F}^{-1}\left\{\log \left(|\mathcal{F}\{f(t)\}|^{2}\right)\right\}\right|^{2} .
$$

The free variable has the dimension of time and has been coined 'quefrency' (comically in line with the rest of the cepstrum vocabulary). The periodicity can be obtained by taking the inverse of it.

Noll and Schroeder proposed the short-time cepstrum version for their speech research [5]. We can construct the short-time cepstral graph, or 'cepstrogram' from the discrete STFT through,

$$
\operatorname{CG}\{x[n]\}(m, k)=\left|\operatorname{IFFT}\left\{\log \left(|\operatorname{STFT}\{x[n]\}(m, k)|^{2}\right)\right\}\right|^{2}
$$

The cepstrogram will prove particularly valuable in the case of long integration interval measurements on rotor resp. propeller carrying targets. We can use it to determine the spectrogram periodicity, which is related to the blade flash frequency, which in turn is related to the angular velocity of the rotor or propeller. In case of multiple rotors, we might even determine whether we deal with a single rotor, or multicopter type target. In clear cases, we can even estimate the number of rotors and their individual angular velocities. To illustrate this, we have generated synthetic time signals of mini-UAVs having $1,2,4,6$ and 8 rotors. We assumed $12 \mathrm{~cm}$ blade length with 2 blades per rotor. The average rotation rate was 80 RPS, with 6 RPS difference between the rotors. In addition, we have added a slight 3 RPS variance on the rotors to account for some minor steering dynamics over time. The simulations were done for a $\mathrm{CW}$ radar operating at $10 \mathrm{GHz}$, with a sampling rate of $24 \mathrm{kHz}$ and $N / f_{s}=85.3 \mathrm{~ms}$.

Fig. 3 and Fig. 4 show the cases for a single rotor and 6 rotors respectively. Note that the cepstrum peaks around $6.25 \mathrm{~ms}$, which indicates a periodicity of $160 \mathrm{~Hz}$ originating from the 2 blade flashes we see for each full rotor cycle. Also note the 6 lines in Fig. 4 and their non-equidistant position in the cepstrogram, due to the different rotation rates.
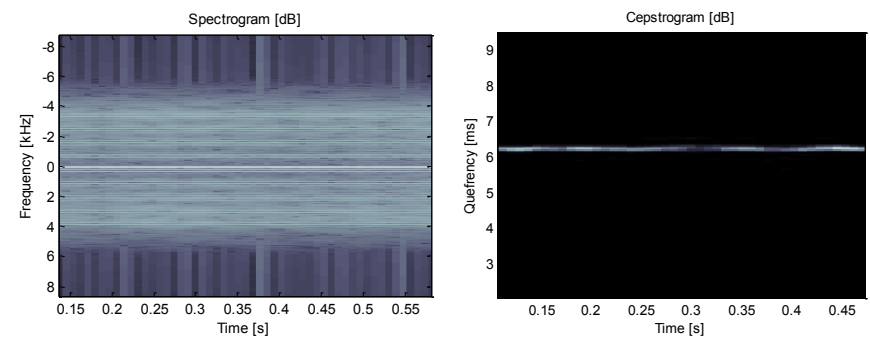

Fig. 3. Spectrogram and cepstrogram from a simulated stationary target with 1 rotor carrying 2 blades.
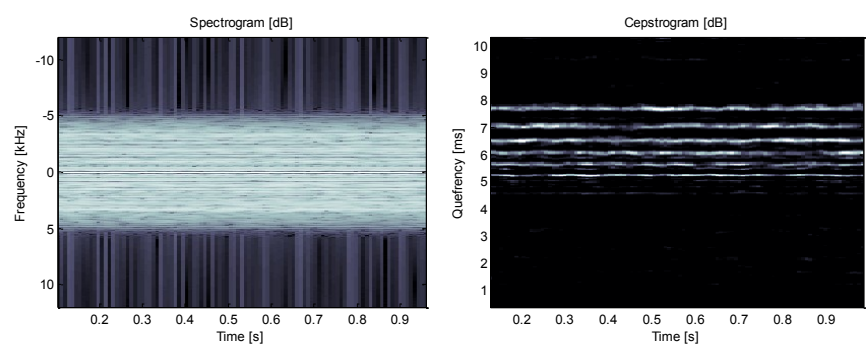

Fig. 4. Spectrogram and cepstrogram from a simulated stationary target with 6 rotors carrying 2 blades per rotor.

With 3 blades per rotor, we would have found the cepstral peaks around $4.17 \mathrm{~ms}$.

To further demonstrate the potential of the cepstrogram, we point out the natural cyclic property of the (inverse) Fourier transform in (3). Let us assume a radar with a sampling rate of only, say $3 \mathrm{kHz}$, corresponding to a feasible range-Doppler surveillance operation. The blind velocity at X-band is around 
$+/-22.5 \mathrm{~m} / \mathrm{s}$, whilst the blade tip velocity in our example is $2 \pi \cdot 12 \cdot 10^{-2} \cdot 80=60.3 \mathrm{~m} / \mathrm{s}$, so significant aliasing occurs. This disables us to find the micro-Doppler spectrum width. However, if we monitor the cepstrogram, we can still identify the micro-Doppler periodicity of the target, indicating a manmade object, probably a mini-UAV. This is illustrated in Fig. 5 for a quadcopter type target. Note that the cepstral resolution is much lower now, but still shows multiple rotors.
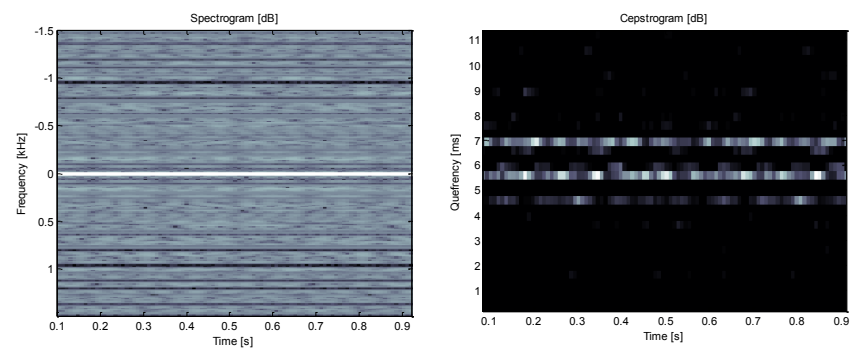

Fig. 5. Spectrogram and cepstrogram from a simulated stationary target with 4 rotors and 2 blades per rotor. Sampling frequency is $3 \mathrm{kHz}$.

\section{APPLICATION ON REAL MEASUREMENTS}

A low power $\mathrm{CW}$-radar operating at $\mathrm{X}$-band was used to acquire measurements on real life targets. In Fig. 6 a measurement on a small radio-controlled (RC) helicopter is shown. The spectral width is clearly visible in the long integration interval and appears quite constant. The short integration interval reveals a relative slowly rotating, evenbladed main rotor and a much faster rotating, yet shorter second rotor (the tail rotor), also even-bladed. Both rotors are also visible in the cepstrogram, with the main rotor producing the strongest signal at $20.6 \mathrm{~ms}$ quefrency, which corresponds to $\sim 24$ RPS for the two-bladed main rotor. This value is true for this particular $\mathrm{RC}$ helicopter.
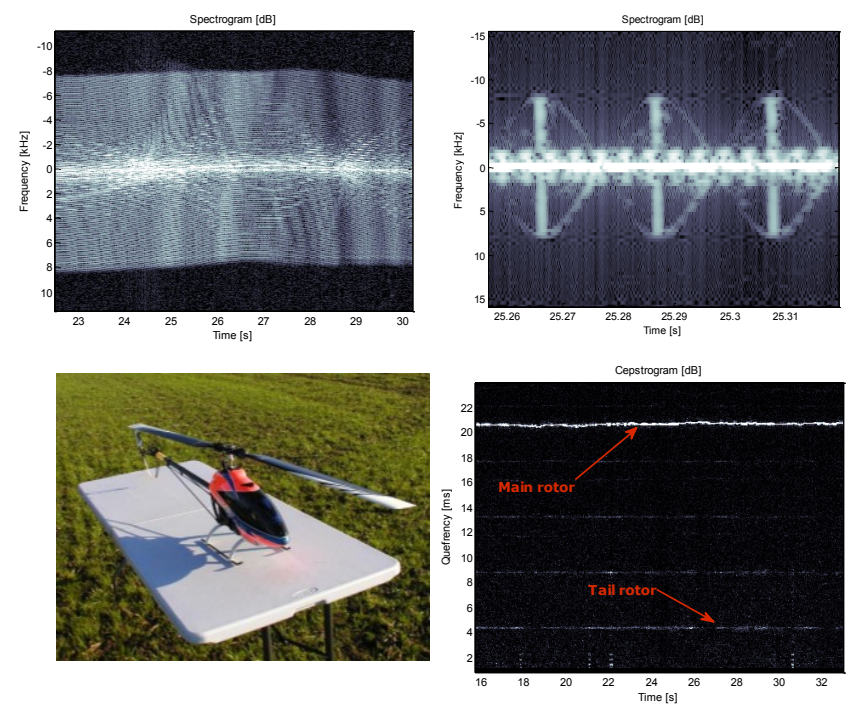

Fig. 6. Long and short integration interval spectrograms (top) of an RC helicopter shown bottom left. The cepstrogram is shown bottom right.

An octocopter example is shown in Fig. 7. The short integration interval now appears chaotic due to the superposition of 8 asynchronous rotors. The long integration interval does reveal the spectral width and quite some dynamics in the separate harmonics, but the periodicity cannot be deduced from the spectrogram. The cepstrogram however show quefrency peaks around $5.5 \mathrm{~ms}$, which is 90.1 RPS for two-bladed rotors. As with the previous example, this value is true for this particular octocopter.
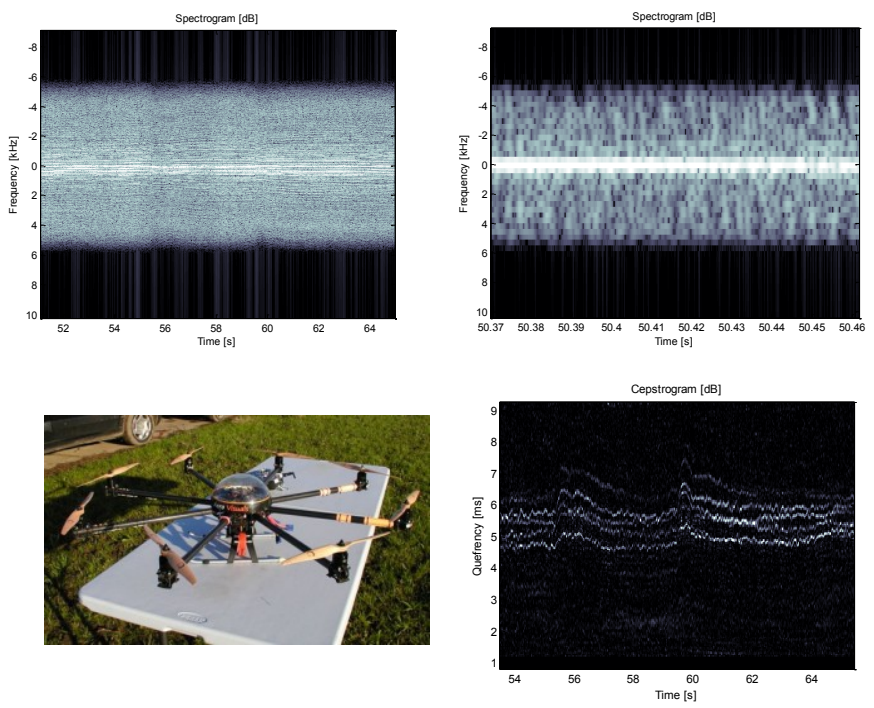

Fig. 7. Long and short integration interval spectrograms (top) of an RC octocopter shown bottom left. The cepstrogram is shown bottom right.

\section{CONCLUSION}

In this paper we have shown that spectrograms and cepstrograms can be used to easily extract key features for automatic or visual recognition of LSS-targets versus bio-life. The long integration interval spectrogram reveals the spectral width, and body velocity. The short integration interval spectrogram shows the spectral symmetry as well as the individual rotor echoes and blade flashes. The cepstrogram shows the periodicity, and in clear cases also the number of rotors. The cepstrogram may also be particularly useful in case of lower sampling frequencies.

\section{ACKNOWLEDGMENT}

This study is performed in the framework of the D-RACE; the Dutch Radar Centre of Expertise, a strategic alliance of Thales Nederland B.V. and TNO.

\section{REFERENCES}

[1] J. J. M. de Wit, R. I. A. Harmanny, and P. Molchanov, "Radar microDoppler feature extraction using the singular value decomposition," submitted to the International Radar Conference 2014.

[2] V. Chen, The Micro-Doppler Effect in Radar, Norwood MA: Artech House, 2011.

[3] J. J. M. de Wit, R. I. A. Harmanny, and G. Prémel-Cabic, "MicroDoppler analysis of small UAVs," in Proc. EuRAD, Amsterdam, The Netherlands, October 31 - November 2, 2012.

[4] B. P. Bogert, M. J. R. Healy en J. W. Tukey (1963): "The Quefrency Alanysis of Time Series for Echoes: Cepstrum, Pseudo Autocovariance, Cross-Cepstrum and Saphe Cracking", in: M. Rosenblatt (ed.): Proceedings of the Symposium on Time Series Analysis, Wiley, New York, Chapter 15, p. 209-243.

[5] A. Michael Noll and Manfred R. Schroeder, "Short-Time 'Cepstrum' Pitch Detection," (abstract) Journal of the Acoustical Society of America, Vol. 36, No. 5, p. 1030. 